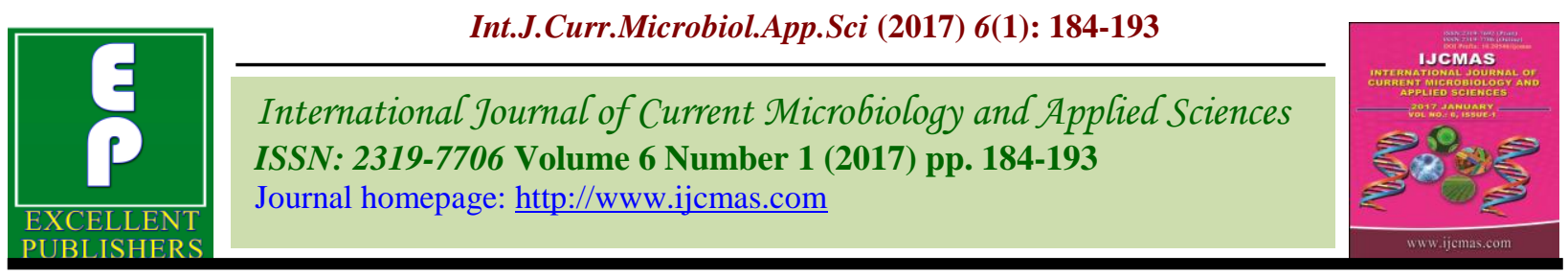

Original Research Article

http://dx.doi.org/10.20546/ijcmas.2017.601.023

\title{
Bacteriological Profile and Antimicrobial Resistance of Blood Culture Isolates from a 350 bedded Hospital Lucknow, India
}

\author{
Manjusha Pandey ${ }^{1}$, Devendra Niranjan ${ }^{2}$ and R.C. Pande ${ }^{3}$ \\ ${ }^{1}$ Department of Microbiology, Vivekananda Polyclinic \& Institute of Medical Sciences, \\ Lucknow, India \\ ${ }^{2}$ Department of Microbiology Career Institute of Medical Science and Hospital Lucknow, India \\ ${ }^{3}$ Department of Microbiology, Saifai Medical College, Etawaa, Uttar Pradesh, India \\ *Corresponding author
}

\section{A B S T R A C T}

\section{Keywords}

Blood Stream

Infection, Antibiotic resistant, Vancomycin Resistant Enterococci, Antimicrobial therapy, Septicemia.

Article Info

Accepted:

12 December 2016

Available Online:

10 January 2017
Blood stream infections can lead to life threatening sepsis and require immediate antimicrobial treatment. Blood culture is an essential tool for the investigation of clinically suspected bacteremia or septicemia. A timely detection of organism type and antibiotic susceptibility testing is critical for the effective treatment of the patient. Illness associated with blood stream infection ranges from self-limiting infections to life-threatening sepsis that require rapid and aggressive antimicrobial treatment. The present study was to describe the profile of bacterial isolates from the blood cultures in a Polyclinic and to know their antibiotic resistance, so that the study can provide guidelines for choosing an effective antibiotic therapy in cases of septicemia. This is a retrospective study of 2452 blood samples collected from clinically suspected cases of bacteraemia reviewed over a period of one year in vevekananda polyclinic \& institute of medical Sciences. 2452 blood samples collected over a year from clinically suspected cases of bacteremia were studied. The isolates were identified by standard biochemical tests and antimicrobial resistance patterns were determined by CLSI guidelines 2013. Positive cultures were obtained in 752 (30.6\%) cases. Among culture positive isolates, Gram-negative bacteria accounted for $51.2 \%$ cases; most common being Klebsiella spp (23\%) followed by Escherichia coli (20\%) and Acinitobacter (04\%). Of the pathogenic Gram-positive isolates, Enterococci (20\%) was the predominant isolate followed by Coagulase negative Staphylococci (16\%). Maximum Gram-negative isolates were sensitive to Colistin (95\%) and Imipenen Meropenem (90). Daptomycin sensitivity was reported in 100\% Staph. aureus and CONS and Vancomycin 90\% in Enterococcus spp. This study provides information on antibiotic resistance of blood isolates. It may be a useful guide for physicians initiating empiric therapy and will help in formulation of antibiotic therapy strategy.

\section{Introduction}

Blood stream infections range from selflimiting infections to life threatening sepsis that requires rapid and aggressive antimicrobial treatment. A wide spectrum of organisms has been described that cause blood stream infections and this spectrum is subject to geographical alteration. Increasing antimicrobial resistance is a worldwide concern. The prevalence of resistance of blood borne isolates is increasing and it also 
varies in accordance with geographical and regional location (Kalpesh et al., 2014)

Blood culture is an essential tool for investigation of clinically suspected bacteremia or septicemia, and guidance for appropriate antimicrobial management (Raghava et al., 2015).

The infection caused by MDR organisms is more likely to prolong the hospital stay, increase the risk of death, and require treatment with more expensive antibiotics. In almost all cases, antimicrobial therapy is initiated empirically before the results of blood culture are available. Keeping in mind the high mortality and morbidity associated with septicemia, right choice of empiric therapy is of importance. Increasing antimicrobial resistance is a worldwide concern. The prevalence of resistance in hospitalized patients with septicemia is increasing, and it varies in accordance with geographical and region allocation. In almost all cases antimicrobial therapy is initiated empirically before the results of blood culture are available. Keeping in mind the high mortality and morbidity associated with septicaemia, a right choice of empiric therapy is of utmost importance.

The present study was undertaken to analyze the various organisms causing septicemia and their antibiotic resistance patterns, and also prevent the hospital infection. as it would be a useful guide for clinicians initiating the empiric anti-microbial therapy.

\section{Materials and Methods}

In this retrospective study, a total of 2,452 blood samples from the clinically suspected cases of bacteraemia were reviewed for a period of one year from January 2014 to December 2015. All the samples were collected at Vivekananda Polyclinic and
Institute of Medical sciences, 350 bedded, tertiary care, teaching Hospital providing full range of medical, surgical and super speciality facilities. Processing of samples was done at the Department of Microbiology, VPIMS, Lucknow, Uttar Pradesh, India.

Blood was collected from 2 different sites average $5 \mathrm{~mL}$ per site in every patient using strict aseptic precautions by nursing personnel or physician and inoculated immediately into $50 \mathrm{ml}$ of 'Brain Heart Infusion' (BHI) broth with $0.025 \%$ of sodium polyanethol sulphonate as anticoagulant (Bactec Blood culture bottle).

BacT/ALERT FA plus-aerobic blood culture bottle for adult patients and in pediatric cases 1-2 mL of blood was inoculated in BacT/ALERT PF plus culture bottles. After collection these bottles were immediately incubated in BacT/ALERT 3D (manufactured by bioMerieoux) — a fully automated blood culture system for detection of growth in blood culture. The negative results were followed up to 5 days and final report was issued. While, in case of a positive growth, the BacT/ALERT automatically gives an alert. After the positive indication the sample were sub cultured on 5\% sheep blood agar and MacConkey agar and put for overnight incubation.

After growth the colonies on blood agar 0.5 McFarland suspension was prepared which was then subjected to identification and susceptibility testing on Vitek 2 (manufactured by bioMerieoux) - which is a fully automated system for identification of organism and antimicrobial susceptibility testing as per the CLSI 2013 guidelines. The card was automatically filled by a vacuum device, sealed and inserted into the VITEK 2 reader-incubator module (incubation temperature, $35.5^{\circ} \mathrm{C}$ ), and subjected to a kinetic fluorescence measurement every 15 
min. The results were interpreted by the IDGPC database, and final results were obtained automatically. All cards used were automatically discarded into a waste container.

The ESBL status was determined by Vitek 2 as per the CLSI guidelines and was not subjected to any further testing.

\section{Data Analysis}

Data was analyzed as per the CLSI 2013 guidelines. which were received in the clinical microbiology laboratory during the period. The patient's name, accession number, date and number of bottles received, earliest date of positive results, number of positive bottles, and final organism identification were recorded and evaluated.

\section{Results and Discussion}

During the one year study period 2452 blood cultures were cultured. $1700(69.3 \%)$ blood culture show no any growth. 752(30.6\%) positive growth were isolated from patients. Most blood stream infection were due to a single organism, while $20(2.6 \%)$ were of polymicrobial aetiology. In 11 of these episodes, two different microbes were detected while in 4 patients three microbes were isolated. All the polymicrobial infections were isolated from hospital admitted patients. Gram-negative bacteria were encountered more often 385(37.8\%) than Gram-positive micro-organisms. The common Gram-negative organisms were Klebsiella spp 173(23\%) followed by Escherichia coli 152(20.2\%), Acinetobacter spp 30(4\%), Enterobacter and Salmonella typhi $15(2 \%)$ and pseudomonas 6(0.7\%).

Among the Gram-positive bacteria, Enterococcus spp. was the predominant isolate $157(20.8 \%)$ followed by a Coagulase -negative Staphylococcus 120(16\%) and Staphylococcus aureus 61 (8.1\%). The distribution of bacterial species of the 752 microorganism isolated Table- 1 .

The $S$. aureus strains showed resistance to vancomycin in $4(0.5 \%)$ isolates. In Enterococcus spp., resistance to vancomycin was seen in $10(1.3 \%)$ isolates.

Among Gram positive bacteria Daptomycin shows $100 \%$ sensitivity in all staphylococci spp. followed by vancomycin, teicoplanin and tigcyclin $96 \%$ sensitive (Table 2)

The most common bacterial isolate was Klebsiella spp. Colistin, Imipenem/ Meropenem and ciprofloxacin followed by pipracillin tazobactum were seen very effective drug with low resistance of $8 \%, 10 \%, 27 \%$ and $30 \%$ respectively. In Escherichea coli, Colistin, imepenem/ meropenem and amikacin was seen low resistant 5\%. 6\% and $12 \%$ respectively. In Acinetobacter imepenem/ meropenem show lower resistant $10 \%$ followed by cefaparazone and clarithromycin $12 \%$. Entrobacter spp show lower resistant with Imipenem/Meropenem (8\%), salmonella with Gentamycin (2\%) and Pseudomanas in Clarithromycin $4 \%$.

Among Gram negative bacteria Amoxicillin noted as high resistant drug and Imepenem/ meropenem in some bacteria colistin was seen as a most sensitive drug (Table 3 )

Fungal septicemia caused by Candia albicans isolated $03 \%$ in total no of samples.

We isolated the bacteria most causing septicemia and their susceptibility pattern with the distribution of the microorganism in this retrospective study from hospital blood samples. The incubation period employed for automated continuous-monitoring blood 
culture systems, when introduced, was six to seven days. The currently recommended incubation period is now commonly accepted as five days. In this study, we reviewed the time to positivity for 2452 blood cultures over a period of one year. We have shown that more than 90 percent of organisms from blood cultures were detected within three days of incubation. This is almost identical to the previous blood culture study reported by others (Doern, 2000). At least one study has shown that 97 percent of significant isolates were recovered within three days of incubation (Bourbeau et al., 2001).

Only 752 organisms out of a total of 2,452 were isolated after the third day of incubation. Review of clinical information for days four and five isolates demonstrated that they were of limited clinical significance. Similar studies using different automated blood culture systems have shown that three or four days of incubation may be sufficient to detect clinically significant organisms.( Johnson et al., 2000). It is in accordance with the studies of China and Gupta (2013), Kamga et al., (2011), Anbumani et al (2008), and Karlowsky et al (2004) who reported similar incidencesbut in most of the studies like, Mehdinejad et al (2007), Barati et al (2009), Ayobola et al (2011) and Mehta et al (2005).

In view of significant vancomycin resistance of $0.5 \%$, in staphylococcal isolates, drugs like daptomycin, tigcyclin and teicoplanin should be considered in the treatment of MRSA . Vancomycin resistant Enterococcus (VRE) in our study is $1.3 \%$ which is in accordance with the studies like Gupta et al., (2015) reported 1.3 to $4 \%$

Enterococcus spp. was the predominant isolate $20.8 \%$ cases in this present paper. The isolation of Enterococcus is the consist with the study with the contradictory result kalpesh et al., $3.5 \%$ (2014) in this study
Coagulase-negative Staphylococcus (16\%) isolated it may be due to skin contamination similar studies Sapna et al., (2015) while the target rates for the contamination should ideally not exceed $2 \%-3 \%$. The actual rates for the contamination vary widely from institution to institution, ranging from $0.6 \%$ to over $6 \%$ (1997). Studies which were done in Virginia (USA) and south India also showed that these organisms, particularly CONS, were an increasing source of true bacteraemia, especially in patients with prosthetic devices and central venous catheter. Therefore, it is extremely important that certain criteria are set for determining the contamination in the blood cultures.

Staphylococcus was isolated in $8 \%$ of cases and CONS in $16 \%$ of cases in the present paper. The isolation of Staphylococcus aureus is consistent with the study of Arora and Devi, Roy et al., (2002), and Karlowsky et al., (2004) where the reported isolation of the organism was $27.3 \%, 14 \%$, and $16.5 \%$, respectively. However, reported isolation of CONS was $20.16 \%, 16.5 \%$, and $42 \%$, respectively, similar to our study. CONS isolated from blood are often skin contaminants which are clinically insignificant.

Klebsiella $23 \%$ Escherichia coli $20.2 \%$ was the most frequent Gram negative pathogen isolated in this present study that is similar to study of Mehta et al., Karlowsky et al., Kamga et al., (2011), and China and Gupta (2013).

In this study also observed frequency of Acinetobacter as in this study that is $4 \%$ but we observed only $2 \%$ Enterobacter, Salmonella and $0.7 \%$ pseudomonas. This is isolated in the frequency of 10 to $20 \%$ in other studies. Generally, Salmonella is community-acquired infection in general population which gains entry via feco-oral route (2004). 
The most common bacterial isolate was Klebsiella spp. Colistin, Imipenem/ Meropenem and ciprofloxacin followed by pipracillin tazobactum were seen very effective drug with low resistance of $8 \%, 10 \%, 27 \%$ and $30 \%$ respectively. High resistant Klebsiella with seen in trimethoprim sulfamethoxazole followed by amoxicillinclavulanate and ceftrioxane $80 \%, 75 \%$ and 73 $\%$ respectively.
In Escherichea coli, Colistin, imepenem/ meropenem and amikacin was seen low resistant $5 \% .6 \%$ and $12 \%$ respectively. In Acinetobacter imepenem/ meropenem show lower resistant $10 \%$ followed by cefaparazone and clarithromycin $12 \%$. Entrobacter spp show lower resistant with Imipenem/Meropenem (8\%), salmonella with Gentamycin (2\%) and Pseudomanas in Clarithromycin $4 \%$.

Table.1 Spectrum of microorganism in blood culture from all wards

\begin{tabular}{|l|l|c|c|}
\hline Sn.no. & Isolated Organism & $\begin{array}{c}\text { Total no of organism } \\
(\mathrm{n}=752)\end{array}$ & Percent (\%) \\
\hline 1. & Klebsiella spp. & 173 & $23 \%$ \\
\hline 2. & Enterococci & 157 & $20 \%$ \\
\hline 3. & Escherichieae coli & 152 & $20 \%$ \\
\hline 4. & CONS & 120 & $16 \%$ \\
\hline 5. & Staph.aureus & 61 & $08 \%$ \\
\hline 6. & Acinetobacter & 30 & $04 \%$ \\
\hline 7. & Pseudomonas & 6 & $0.8 \%$ \\
\hline 8 & Salmonella spp. & 15 & $02 \%$ \\
\hline 9. & Enterobacter & 15 & $02 \%$ \\
\hline 10. & Candia albicans & 23 & $03 \%$ \\
\hline
\end{tabular}

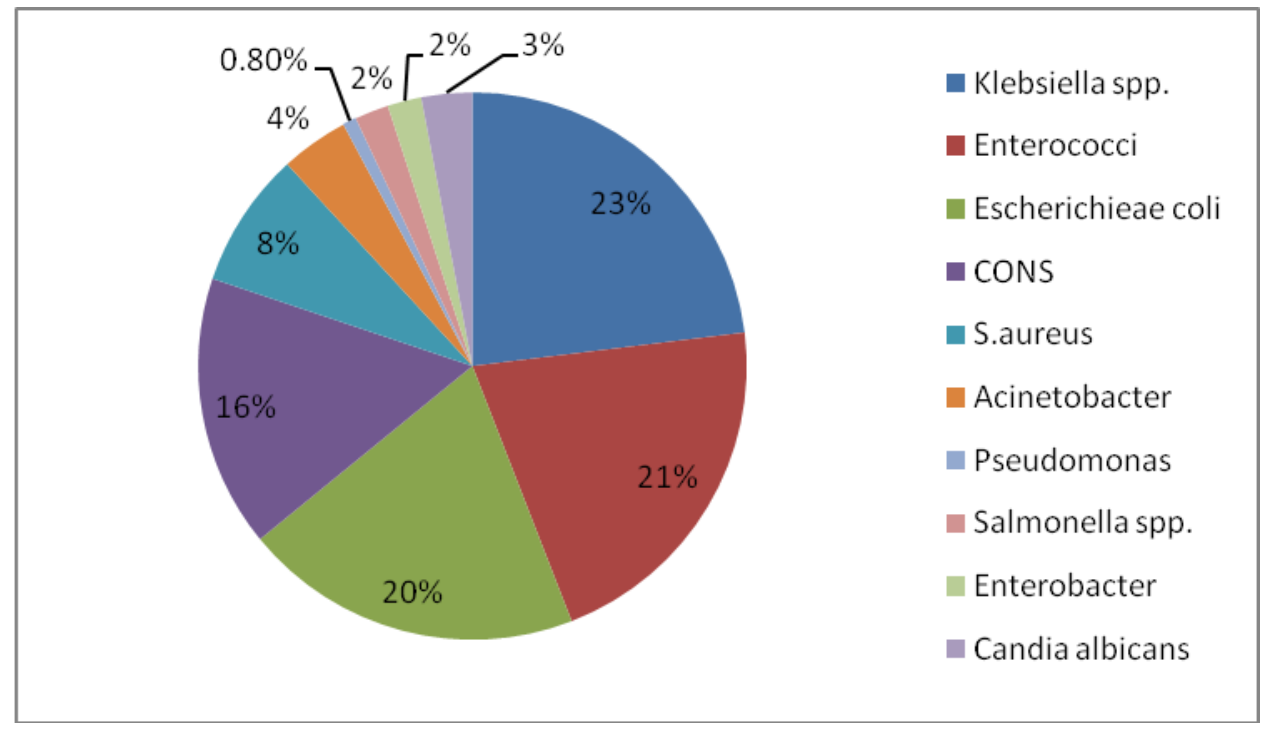


Table.2 Resistant pattern of Gram Positive organism ( $\mathrm{n}=338$ )

\begin{tabular}{|l|l|l|l|l|l|l|l|l|l|l|}
\hline Organism & Amp & $\begin{array}{l}\text { Lino } \\
\text { zo }\end{array}$ & $\begin{array}{l}\text { Vanc } \\
\text { o }\end{array}$ & $\begin{array}{l}\text { Teico } \\
\mathrm{p}\end{array}$ & $\begin{array}{l}\text { Doxy/te } \\
\text { tra }\end{array}$ & $\begin{array}{l}\text { Cipr } \\
\text { o }\end{array}$ & $\begin{array}{l}\text { Gent } \\
\text { a }\end{array}$ & Levo & $\begin{array}{l}\text { Dap } \\
\text { to }\end{array}$ & $\begin{array}{l}\text { tigcy } \\
\text { clin }\end{array}$ \\
\hline CONS $(n=120)$ & 90 & 5 & 8 & 7 & 20 & 25 & 28 & - & 0 & 8 \\
\hline S.aureus $(n=61)$ & 90 & 88 & 4 & 4 & 15 & 30 & 32 & - & 0 & 4 \\
\hline $\begin{array}{l}\text { Enterococci }(n=1 \\
\text { 57) }\end{array}$ & 90 & 12 & 10 & 10 & 38 & 28 & 87 & 30 & - & - \\
\hline
\end{tabular}

Amp=Ampicillin,Lino=Linozolid,Teico=Tecoplanin,Doxy=Doxycyclin,Tetra=tertracyclin,Cipro $=$ Ciproflox, Genta $=$ Gentamycin,Levo=Levoflox,Dapto=Dapyomycin,Tig=Tigcyclin

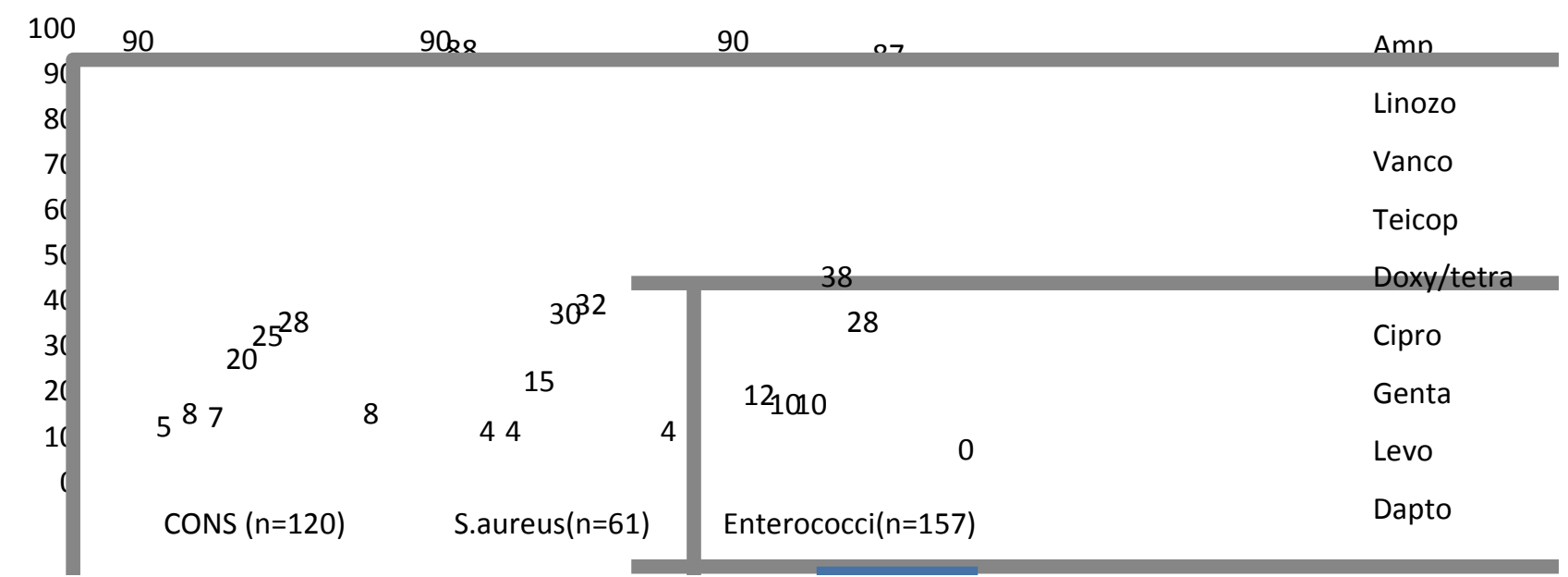

Table.3 Resistant pattern of Gram Negative organism ( $\mathrm{n}=385)$

\begin{tabular}{|l|c|c|c|c|c|c|}
\hline & $\begin{array}{c}\text { Escherichea } \\
\text { coli }(n=152)\end{array}$ & $\begin{array}{c}\text { Klebsiella } \\
(n=173)\end{array}$ & $\begin{array}{c}\text { Pseundomonas } \\
N=6\end{array}$ & $\begin{array}{c}\text { Salmonella } \\
(n=15)\end{array}$ & $\begin{array}{c}\text { Acinitobactor } \\
(n=30)\end{array}$ & $\begin{array}{c}\text { Entnerobacter } \\
(n=15)\end{array}$ \\
\hline Colistin & $5 \%$ & $8 \%$ & - & - & - & - \\
\hline IMP/MEM & $6 \%$ & $10 \%$ & $6 \%$ & $0 \%$ & $10 \%$ & $8 \%$ \\
\hline Ertapenem & $48 \%$ & $55 \%$ & - & - & - & - \\
\hline Pip/tazo & $22 \%$ & $30 \%$ & $10 \%$ & - & $20 \%$ & $22 \%$ \\
\hline CFS & $35 \%$ & $65 \%$ & $10 \%$ & $40 \%$ & $12 \%$ & $15 \%$ \\
\hline Cipro/oflox- & $40 \%$ & $27 \%$ & $45 \%$ & - & $25 \%$ & - \\
\hline Amika & $12 \%$ & $42 \%$ & $25 \%$ & - & $33 \%$ & \\
\hline Ceftraxone & $62 \%$ & $73 \%$ & - & - & - & - \\
\hline Tmp/SMX & $65 \%$ & $80 \%$ & - & $30 \%$ & & \\
\hline Amoxi/clave & $70 \%$ & $75 \%$ & - & $40 \%$ & $95 \%$ & \\
\hline Clarithro & - & - & $4 \%$ & $0 \%$ & $12 \%$ & \\
\hline Genta & - & - & - & $20 \%$ & - & - \\
\hline Ceftizidime & - & - & $40 \%$ & & & \\
\hline Azreonam & & & $65 \%$ & - & $58 \%$ & \\
\hline
\end{tabular}

Imp=impenem,Mem=Meropenem,Pip=Pipracyclin,Tazo=Tazobactum,CFS=Cefaparazone,Cipro $=$ Ciproflox, Oflox $=$ Ofloxacin,Amika=Amikacin, $\mathrm{Tmp}=$ Trimethoprim, $\mathrm{Smx}=$ sulfamethoxazole, $\mathrm{Cla}$ rithro=Clarithromycin, Genta=Gentamycin 
Fig 1 Showing Antibiotic Resistant Pattern of Escherichia coli and Klebsiella spp.

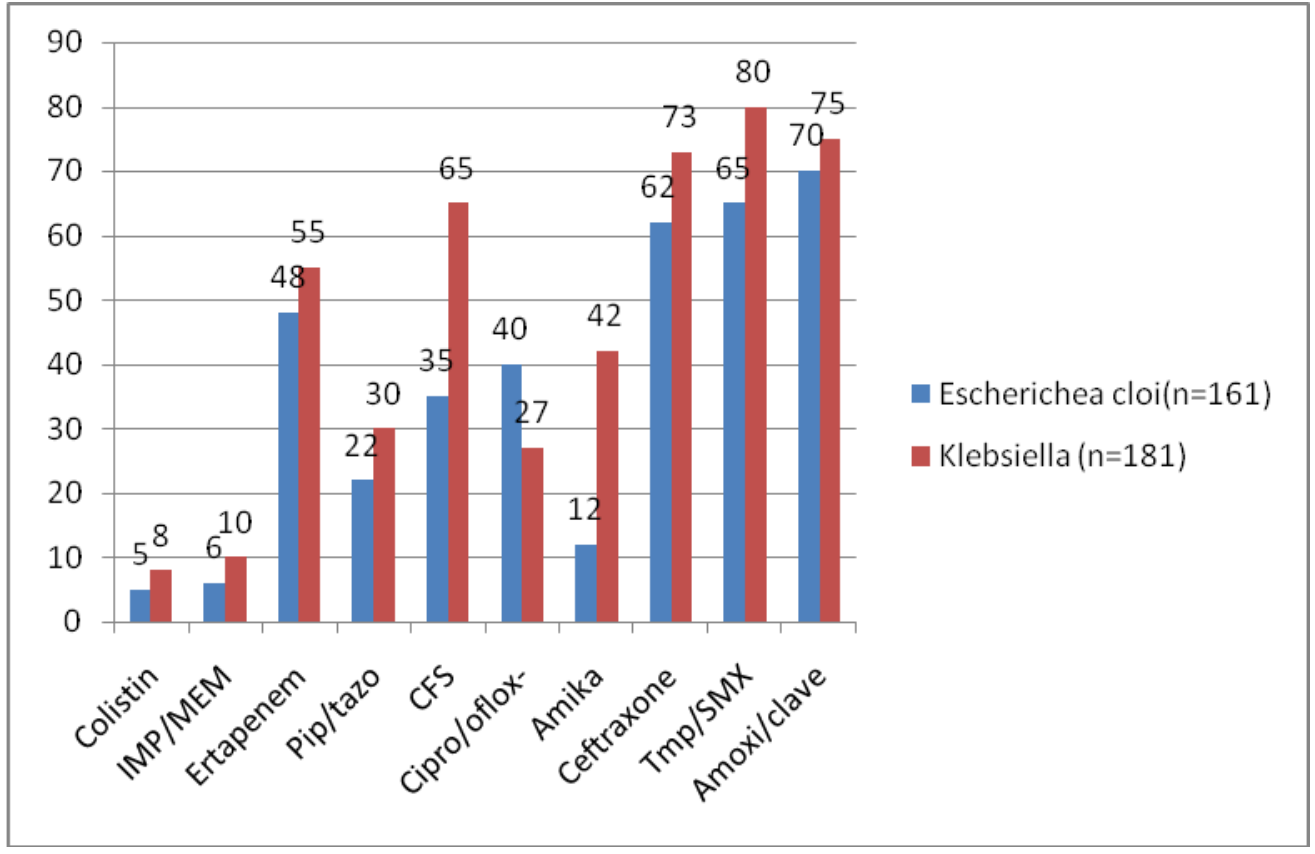

Fig 2 Showing Antibiotic Resistant Pattern of Psedumonas and Salmonella spp

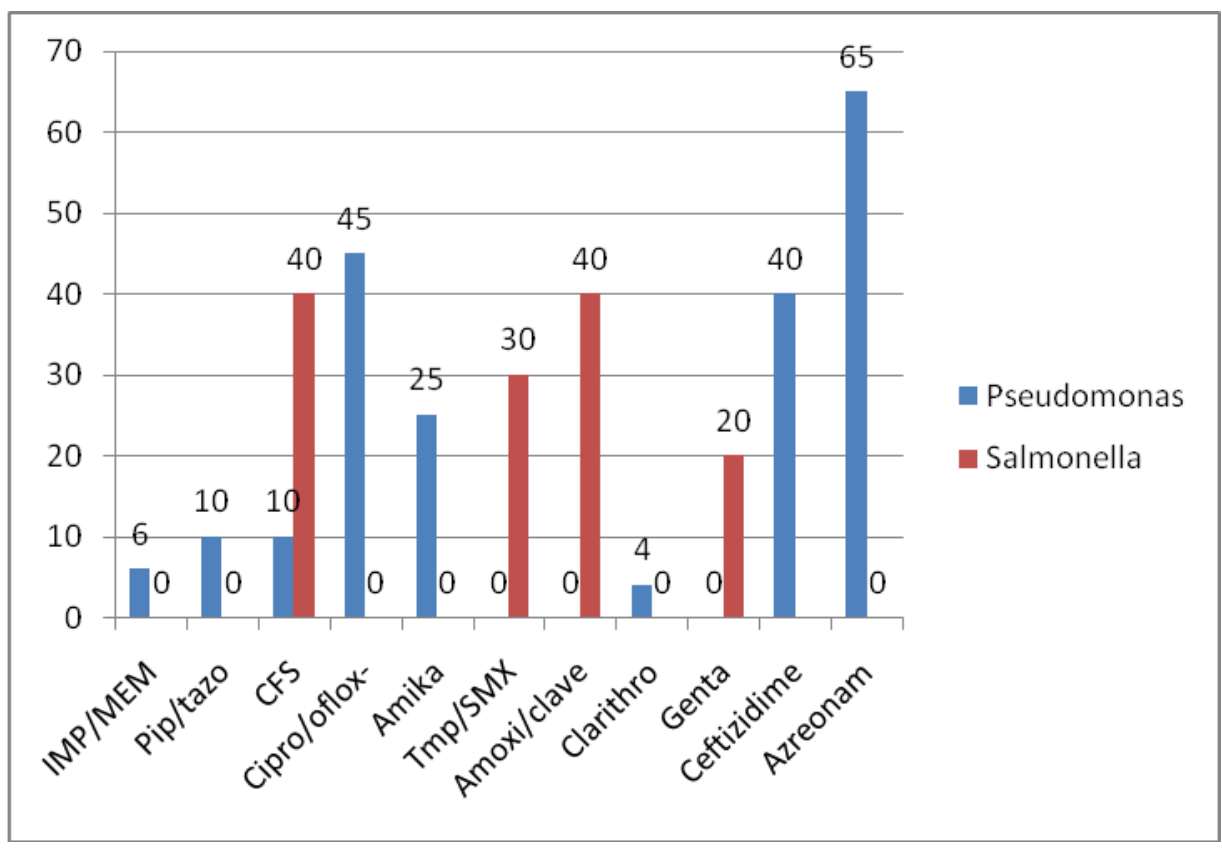


Fig 3 Showing Antibiotic Resistant Pattern of Acinetobacter and Enterobacter spp.

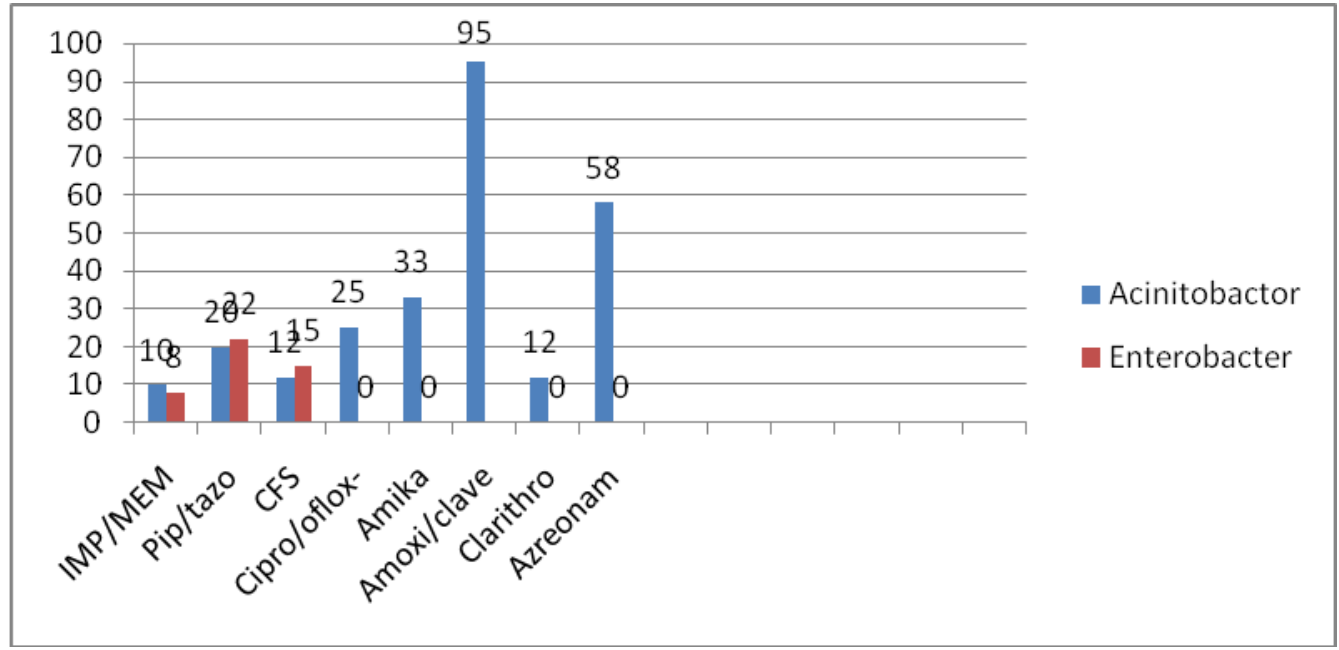

Among Gram negative bacteria Amoxicillin noted as high resistant drug and Imepenem/ meropenem in some bacteria colistin was seen as a most sensitive drug similar to study of Kavitha Prabhu et al., (2010).

The $S$. aureus strains showed resistance to Ampicillin followed by ciprofloxacin and gentamycin, $80 \%, \quad 32 \%$ and $30 \%$ respectively. Vancomycin resistant found in $4(0.5 \%)$ isolates. In Enterococcus spp., resistance to vancomycin was seen in $10(1.3 \%)$ isolates (Table 2 )

Among Gram positive bacteria Daptomycin shows 100\% sensitivity in all staphylococci spp. followed by vancomycin, teicoplanin and tigcyclin 96\% sensitive. Similar study observed to prakash et al., (2011) \& Mehta et al., (2005).

In this current study fungal infection caused by Candida albicans observed $03 \%$ that is found more than few gram negative bacteria.

In conclusion, this data provided important information on the prevalence of antimicrobial resistance amongst pathogens causing blood stream infections. BSI an important cause of morbidity and mortality in our patients especially among chronically ill elderly adult males. Coagulasenegative staphylococcus, klesiellaspp and E. coli were the most important bacterial pathogens causing BSI. Prescription of Trimethoprim/ Sulphamethoxazole, Ampicillin, Cepharadine, Gentamicin and Amoxicillin/Clavulanic acid to bacteremia patients needs attention. The rise in antibiotic resistance in blood isolates emphasizes the importance of sound hospital infection control, rational prescribing policies, and the need for awareness to use antimicrobial drugs. Our results seem helpful in providing useful guidelines for choosing an effective antibiotic in cases of septicaemia and for choosing salvage therapy against BSI and also to determine the source of infection and risk factors associated with BSI are further considered necessary.

\section{References}

Kalpesh Gohel, Amit Jojera, Shailesh Soni et al. 2014. Bacteriological Profile and Drug Resistance Patterns of Blood Culture Isolates in a Tertiary Care Nephrourology Teaching Institute. Bio Med. Res. Int., vol 2, pp 267-272. 
Raghava Potula, Vipul Dadhania, Allan, L. 2015. Truant Automated blood culture testing: A retrospective study indicates that a three-day incubation period is sufficient. The Peer-Reviewed Management Source for Lab Professionals September, 2015

Garg, A., S. Anupurba, and J. Garg. 2007. "Bacteriological profile and antimicrobial resistance of blood culture isolates from a university hospital," J. Indian Academy of Clin. Med., vol. 8, no. 2, pp. 139-143.

Doern, G.V. 2000. Detection of selected fastidious bacteria. Clin. Infect. Dis., 30: 166-173.

Doern, G.V., Brueggemann, A.B., Dunne. W.M., Jenkins, S.G., Halstead, D.C., McLaughlin, J.C. 1997. Four-day incubation period for blood culture bottles processed with the Difco ESP blood culture system. J. Clin. Microbiol., 35: 1290-1292.

Bourbeau, P.P., Pohlman, J.K. 2001. Three days of incubation may be sufficient for routine blood cultures with BacT/Alert FAN blood culture bottles. J. Clin. Microbiol., 39: 2079-2082.

Johnson, A.S., Touchie, C., Haldane, D.J., Forward, K.R. 2000. Four-day incubation for detection of bacteremia using the BACTEC 9240. Diagn Microbiol Infect Dis., 38: 195-199.

China, V. Gupta. 2013. "Bacteriological profile and antimicrobial susceptibility pattern of blood isolates from a tertiary care hospital in North India," Int. J. Pharmaceutical Res. Biosci., vol. 2, no. 2, pp. 24-35.

Kamga, H.L.F., A.L. Njunda, and P.F. Nde. 2011. "Prevalence of septicemia and antibiotic sensitivity pattern of bacterial isolates at the University Teaching Hospital, Yaoundae, Cameroon," African J. Clin.
Experimental Microbiol., vol. 12, no. 1, pp. 2-8.

Anbumani, N., J. Kalyani, and M. Mallika. 2008. "Distribution and antimicrobial susceptibility of bacteria isolated from blood cultures of hospitalized patients in a tertiary care hospital," Indian J. Practicing Doctor, vol. 5, no. 2, pp. 17.

Karlowsky, J.A., M.E. Jones, D.C. Draghi, C. Thornsberry, D.F. Sahm, and G.A. Volturo. 2004. "Prevalence and antimicrobial susceptibilities of bacteria isolated from blood cultures of hospitalized patients in the United States in 2002," Annals of Clin. Microbiol. Antimicrobials, vol. 3, article 7.

Mehdinejad, M., A.D. Khosravi, and A. Morvaridi. 2007. "Study of prevalence and antimicrobial susceptibility pattern of bacteria isolated from blood cultures," J. Biol. Sci., vol. 9, no. 3, pp. 249-253.

Barati, M., M.T. Taher, R. Abasi, M.M. Zadeh, et al. 2009. "Bacteriological profile and antimicrobial resistance of blood culture isolates," Iranian J. Clin. Infect. Dis., vol. 4, no. 2, pp. 87-95.

Ayobola, E.D. O.S. Egbule, and O. Omonigho. 2011. "Study of prevalence and antimicrobial susceptibility of blood culture bacterial isolates," Malaysian J. Microbiol., vol. 7, no. 2, pp. 78-82, 2011.

Mehta, P. Dutta, and V. Gupta. 2005. "Antimicrobial susceptibility pattern of blood isolates from a teaching hospital in North India," Japanese J. Infect. Dis., vol. 58, no. 3, pp. 174-176.

Vrasha Gupta, nidhi Singla, et al. 2015. Antimicobial susceptibility patteren of Vancomycin resistant enterococci to newer antimicrobial agents", The Indian $J$. medical research volume 141,issue 4, page 483-486. 
Kalpesh Gohel, Amit Jojera, Shailesh Soni. 2014. Bacteriological Profile and Drug Resistance Patterns of Blood Culture Isolates in a Tertiary Care Nephrourology Teaching Institute BioMed Res. Int., Volume 2014, Article ID $153747, \quad 5$ pages http://dx.doi.org/10.1155/2014/153747

Sapna Malik, K. Ravishekhar. 2015. Significance of Coagulase Negative Staphylococcus Species in Blood Culture J. Clin. Diagnostic Res., Year Volume : 6 Issue : 4 Page : 632 - 635

Hall, K.K., Lyman, J.A. 2006. Updated review of blood culture. Clin. Microbiol., 19(4): 788-802.

Chandrasekar, P.H., Brown, W.J. 1994. Clinical issues of blood cultures. Arch. Internal Med., 154 : 841-49.

Weinstein, M.P., Towns, M.L., Quartery, S.M., Mirrett, S., et al. 1997. The clinical significance of positive blood cultures in the1990s: a prospective comprehensive evaluation of the microbiology, epidimeology and the outcome of bacteremia and fungemia in adults. Clin. Infect. Dis., 24: 584-602.

Dias, E., Vigneshwaran, P. 2010. The bacterial profile of neonatal septicaemia in a rural hospital in south India. $J$. Clin. Diag. Res., 4(6): 3328-31.

Arora, U. and P. Devi. 2007. "Bacterial profile of blood stream infections and antibiotic resistance pattern of isolates," JK Sci., vol. 9, no. 4, pp. 186190.

Roy, I., A. Jain, M. Kumar, and S. K. Agarwal. 2002. "Bacteriology of neonatal septicemia in a tertiary care hospital of Northern India," Indian J. Med. Microbiol., vol. 20, pp. 156-159.

Karlowsky, J.A., M.E. Jones, D.C. Draghi, C. Thornsberry et al. 2004. "Prevalence and antimicrobial susceptibilities of bacteria isolated from blood cultures of hospitalized patients in the United States in 2002," Annals of Clin. Microbiol. Antimicrobials, vol. 3, article 7, 2004.

Trevini, S. and C.R. Mahon. 2000. "Bacteraemia," in Textbook of Diagnostic Microbiol., R. M. Connie and G. Manusel, Eds., pp. 998-1008

Kamga, H.L.F., A.L. Njunda, and P.F. Nde. 2011. "Prevalence of septicemia and antibiotic sensitivity pattern of bacterial isolates at the University Teaching Hospital, Yaoundae, Cameroon, African J. Clin. Experimental Microbiol., vol. 12, no. 1, pp. 2-8.

Marina de Cueto, Esther Ceballos, Luis Martinez-Martinez. et al. 2004. Use of Positive Blood Cultures for Direct Identification and Susceptibility Testing with the Vitek 2 System. J. Clin. Microbiol., 42(8): 3734-3738.

Kavitha Prabhu, Sevitha Bhat, and Sunil Rao. et al. 2010. "Bacteriologic Profile and Antibiogram of Blood Culture Isolates in a Pediatric Care Unit" J. Lab Physicians, 2(2): 85-88.

Prakash, K.P., Vinod Arora, and Geethanjali, P.P. 2011. "Bloodstream Bacterial Pathogens and their Antibiotic Resistance Pattern in Dhahira Region, Oman. Oman Med. J., 26(4): 240-279.

\section{How to cite this article:}

Manjusha Pandey, Devendra Niranjan and R.C. Pande. 2017. Bacteriological Profile and Antimicrobial Resistance of Blood Culture Isolates from a 350 bedded Hospital Lucknow, India. Int.J.Curr.Microbiol.App.Sci. 6(1): 184-193. doi: http://dx.doi.org/10.20546/ijcmas.2017.601.023 